\title{
Morphological Changes in Keratoconus: Interpretation of Corneal Contocal Microscopy Findings
}

DOI: $10.17691 / \mathrm{stm} 2018.10 .3 .16$

Received February 2, 2017

G.B. Egorova, MD, DSc, Chief Researcher, Department of Refractive Disorders;

A.A. Fedorov, MD, PhD, Head of Fundamental Ophthalmological Research Laboratory;

1.A. Novikov, Senior Researcher, Fundamental Ophthalmological Research Laboratory

Research Institute of Eye Diseases, 11A Rossolimo St., Moscow, 119021, Russia

The aim of the study was to suggest the interpretation of confocal microscopy findings based on their comparison with a corneal morphological picture, and in accordance with the structural principles of confocal images.

Materials and Methods. For 10 year (2005-2015) we monitored and followed up 660 patients with stage I-IV keratoconus (1268 eyes). In addition, 160 patients with subclinical keratoconus (268 eyes) composed a separate group. Corneal discs removed during penetrating keratoplasty were used as the material for paraffin sections to study morphological changes and compare the study results and confocal microscopy findings, confocal microscopy being performed preoperatively. The sections were hematoxylin and eosin stained. To perform corneal confocal microscopy we used a confocal microscope ConfoScan 4 (Nidek Technologies Srl, Japan).

Results and Discussion. The most specific signs of subclinical keratoconus were revealed using corneal confocal microscopy. We suggested the concept for interpreting the signs according to the principles of optics and confocal imaging. An optical phenomenon of increased intensity of light reflection by keratocyte nuclei can be due to the light scattering in surrounding tissues. The phenomenon of visible (needle-shaped) deformity of keratocyte nuclei can result from anomaly of spatial orientation of collagen plates. We suggested an interpretation of the light phenomenon of stria formation in corneal stroma in advanced keratoconus and confirmed it with corneal histology. Corneal defects, fibrocellular proliferation and epithelial proliferation were registered in Bowman's membrane, the findings being confirmed by histological analysis of corneal section. Confocal microscopy revealed possible variants of entocornea defect closure.

Conclusion. The findings enable to assess the informativity of corneal confocal microscopy, provide an interpretation of the results and confirm it using histological analysis of corneal sections.

Key words: keratoconus; corneal morphology; confocal microscopy; interpretation of confocal images.

\section{Introduction}

Corneal morphofunctional status assessment is necessary to achieve success in optical and surgical correction of various refraction disorders, as well as in the evaluation of pathology severity, therapy efficiency, and the determination of patients' management. Currently, there are informative non-invasive research techniques, which enable to visualize the findings that expand both: diagnostic and monitoring capabilities.

One of the methods now used to study cornea is confocal microscopy [1]. It is a variant of optical microscopy that enables to achieve greater amplification and obtain far more contrast images of an object under study compared to standard microscopy images. It can be achieved through the use of aperture situated in the projection plane limiting the background stray light flow. Thus, image contrast enhancement is related to the fact that an illuminator does not provide visual field illuminance through the depth of an image obtained, and focuses light primarily in the focus plane. Most of the converging beams concentrate in a single focal point.
The use of the scheme implies the strict observance of equal sizes of an illuminator aperture and a microscope aperture.

A present-day biological laser scanning confocal microscope at any time registers an image of a single object point, while a WYSIWYG image is made by scanning a sample or by optical system rearrangement [2]. For the first time, a confocal microscope providing high image contrast and a means for examining tissues at cellular level of physiological functioning was suggested by Minsky (USA) in 1961 [3]. Based on a simple optical system, which enables to have a plain optical projection in a monochromatic field light, there was made a confocal microscope for corneal biomicroscopy - ConfoScan 4 (Nidek Technologies Srl, Japan). It makes corneal layerby-layer scanning, the size of the area under study being $440 \times 330 \mu \mathrm{m}$. Both: a central zone and a paracentral zone can be examined.

Currently, the technique is widely applied to diagnose and monitor keratoconus and other corneal dystrophies and diseases. It is possible to assess the condition of corneal tissues and cell elements in this microscopy 
technique when having a certain microscopic picture, imaging being the result of a different degree of light reflection and scattering by structural units of cells and intercellular matrix $[4,5]$.

Despite some depth restrictions for objects exposure, the average brightness of image points is chiefly determined by the nature and intensity of Rayleigh scattering on optical inhomogeneity of corneal tissues. The occurrence of optical inhomogeneities results in light diffraction, the increase of light scattering and average brightness of image points, the size of the optical inhomogeneities exceeding half-wavelength of light. It should not be overlooked that the average brightness of a current shot is influenced by the light scattering in both: cornea over- and under-layers in relation to a viewing plane. It is this placing restrictions on a confocal microscope that used to plot densitometric curves.

The perceived microscopic picture results from a certain combination of structural changes in corneal tissues. However, confocal microscopy specificity as a diagnostic modality of the changes is not high, it is due to the fact that different pathological processes can result in one-type gross variation of refraction, light reflection, and scattering by corneal tissue formed elements.

Currently, there have been gathered large amounts of information on cornea confocal microscopy findings in various pathologies, though not infrequently they are based on suppositions, which are unlikely to be specified and confirmed, since the study is intravital and noninvasive.

However, the image analysis using optical principles, as well as the structural principles of confocal images enables to explain some basic optical phenomena. Partially, the findings can be confirmed by histological findings (the analysis of corneal discs removed during penetrating keratoplasty).

Huge difficulties can be faced when keratoconus is diagnosed at an initial subclinical stage, clinical manifestations missing. In this case, confocal microscopy findings can be supplementary, and in some instances - a cogent argument in favor of the diagnosis. Still, initial changes in keratoconus can be nonspecific as well [6].

The aim of the study was to suggest the interpretation of confocal microscopy findings based on their comparison with a corneal morphological picture, and in accordance with the structural principles of confocal images.

\section{Materials and Methods}

For 10 year (2005-2015) we monitored and followed up 660 patients with stage I-IV keratoconus (1268 eyes). In addition, 160 patients with subclinical keratoconus (268 eyes) composed a separate group. Stage I keratoconus was diagnosed in 320 eyes, stage II keratoconus - in 340 eyes, stage III - in 206 eyes, and stage IV keratoconus - in 134 eyes.
The study complies with the declaration of Helsinki (2013) and was approved by the Ethics Committee of Research Institute of Eye Diseases. All patients gave their written informed consent.

Penetrating keratoplasty was performed on 64 eyes of patients with stages III-IV keratoconus. Corneal discs removed during penetrating keratoplasty were used as the material for paraffin sections, which were hematoxylin and eosin stained. Histological specimens were studied using a light-optical microscope Leica DM2500 (Leica Microsystems, Germany), amplification: 125-400. Morphological changes were compared with preoperative confocal microscopy findings.

In the study, we used a confocal microscope ConfoScan 4.

\section{Results and Discussion}

Generally, the epithelium in subclinical keratoconus is unaltered. In certain cases, there can be initial signs of epitheliopathy in the form of blurring boundaries, impaired intercellular structure, and altered epitheliocyte form. No changes were found in meibomian membrane as well.

One of the earliest structural signs in corneal stroma is the occurrence of keratocyte nuclei with the highest reflectivity (Figure 1). In foreign literature, there are the reports explaining the phenomenon. The increase in keratocyte nuclei brightness is interpreted as the result of cell metabolism growth. Such keratocytes are called activated, stress cells, the activity of which is aimed at maintaining inner corneal homeostasis [1].

The optical phenomenon is interpreted as a response to hypoxia, inflammatory and cicatricial processes, and can be one of early signs of keratoconus: the occurrence of bright keratocyte nuclei is the response to stress [6]. However, optically, the statement is incorrect. To

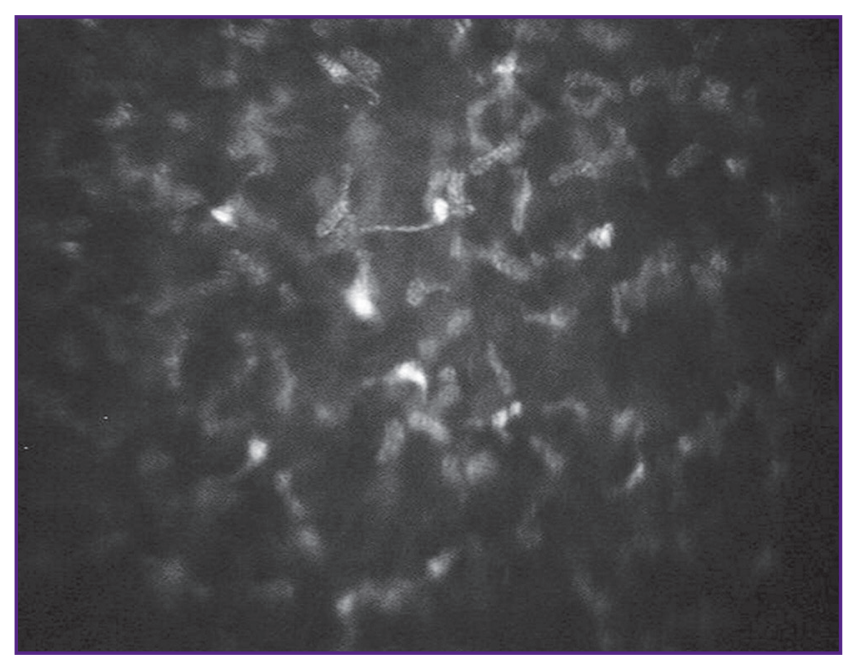

Figure 1. Keratocyte nuclei are in the corneal middle stroma with increased light reflection; FOV $-300 \times 220 \mu \mathrm{m}$ 
increase light reflection it is necessary to enhance the optical density of cell nuclei, and it is inconsistent since the tightening of cell nuclear chromatin is typical for processing proceeding in apoptosis when a cell dies, its metabolism decreasing [7]. Moreover, frequently observed multiple brightness gain of "reflection" at a nuclear membrane surface would be impossible without significant alterations of chemical composition of nuclei or cytoplasm that is certainly prevented by physiological cell stability.

Light reflection changed by stromal cell elements can be explained by the alterations occurring in the structures surrounding a cell, it can be related to distribution of glycosaminoglycans, water imbalance, orientation of stromal collagen lamellae. The alterations occurring lead to reduced tissue transparence, optical homogeneity, and in the end - to light scattering increase.

The main principle of confocal microscopy is the correspondence of a substage lamp aperture size and a microscope aperture that provides the substage lamp ray path length and the beams reflected from an object at an equal angle (Figure $2(\mathrm{a})$ ).
The areas of increased light scattering in corneal stroma ahead of the study object result in the lamp aperture increase (i.e. the angle increase, at which beams converge to the object), it leading to the violation of confocal observance principle and imaginary brightness increase of reflection from the surface of all convex stroma formed elements. In this case, in addition to the lamp beams, which provide certain brightness of convex surfaces, the object being under incident beams from the enlarged zone that enhances the effect of their reflection (Figure 2 (b)).

One of the early signs of morphological changes in corneal stroma revealed in confocal microscopy is the increase of light reflection effect from an endothelial layer in Descemet's membrane. The optical phenomenon occurs in $53 \%$ of cases in subclinical keratoconus (Figure 3 (a), arrow).

An increased light reflection from endothelium can result from enhanced light scattering in the preceding stromal layers.

One more sign should be mentioned, it occurs in subclinical keratoconus - a typical vertical stromal

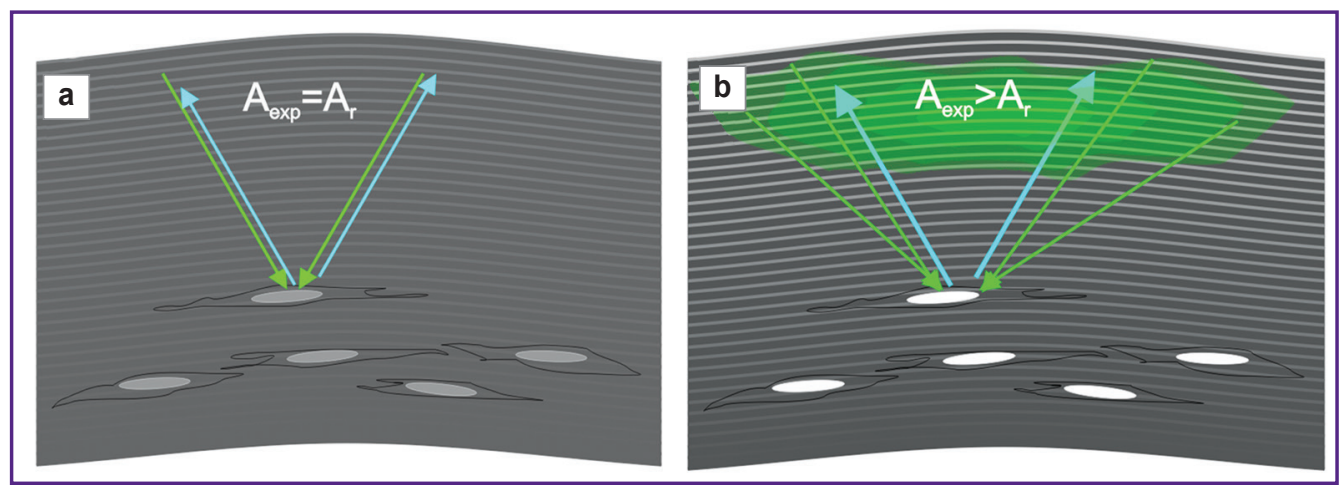

Figure 2. Confocal image formation in the norm (a) and in the enhanced light scattering areas (b)

There has been demonstrated the imaginary increase effect of reflectivity of geometrically convex formed elements in confocal microscopy with an added aperture (b)
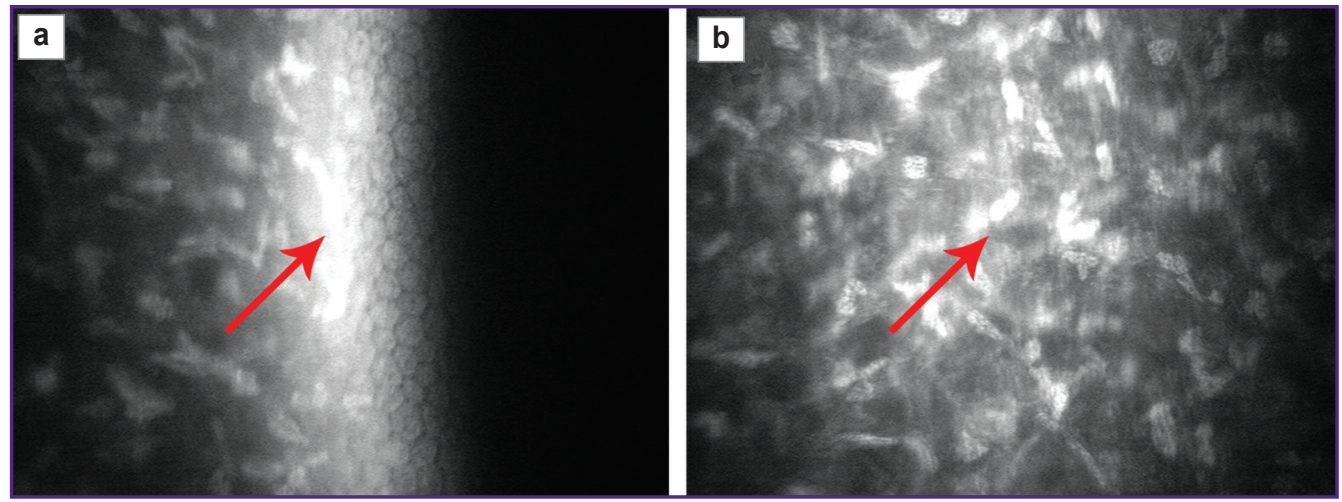

Figure 3. An increased effect of light reflection from the endothelial layer in Descemet's membrane area (arrows):

(a) transverse section; (b) frontal view; FOV - 300×220 $\mu \mathrm{m}$ 
striation with a relevant keratocyte nuclear orientation that is a prognostic of microstria and is more likely related to redistribution of stromal tension lines. The phenomenon occurs in subclinical keratoconus in 30\% of microstria cases and is the most characteristic for keratoconus leading to a clinical stage (Figure 4 (a)).

A specific sign of subclinical keratoconus is an optical phenomenon, which occurs, according to our records, in $57 \%$ cases in posterior stroma layers in the form of needle-shaped formations (Figure 4 (b)). Foreign literature reports about their origin suggesting the ideas about possible alterations of keratocyte nuclei [1]. To our opinion, a phenomenon of a visible keratocyte nuclear deformity can be of optical nature and due to the increment of pathological changes in the corneal stroma, which result in altered spatial orientation of collagen lamellae and the formation of wavy folds. Stromal folds with geometric amplitude increase show the properties of cylinder lens resulting in imaginary spreading of object images due to the bending of reflected beams on fold limbs (Figure 5).

The changes are registered in the posterior stroma layers, though when a pathological process is progressing, some alterations can be revealed in the middle layers as well. Generally, in advanced keratoconus, the sign is missing. The dynamics of morphological impairments indicates a pathological process in keratoconus to start developing in the posterior stromal layers, eventually spreading to the middle stromal layers. The disappearance of needleshaped formations in an advanced disease can be explained by violent structural failures, it concealing the defect in confocal microscopy.

Initial changes in the anterior corneal stroma in stage I keratoconus in some cases can be noticed during biomicroscopy in the forming ectasia apex. There can be seen a grayish area with a non-uniform light reflex this sign is usually described as a symptom of "stroma

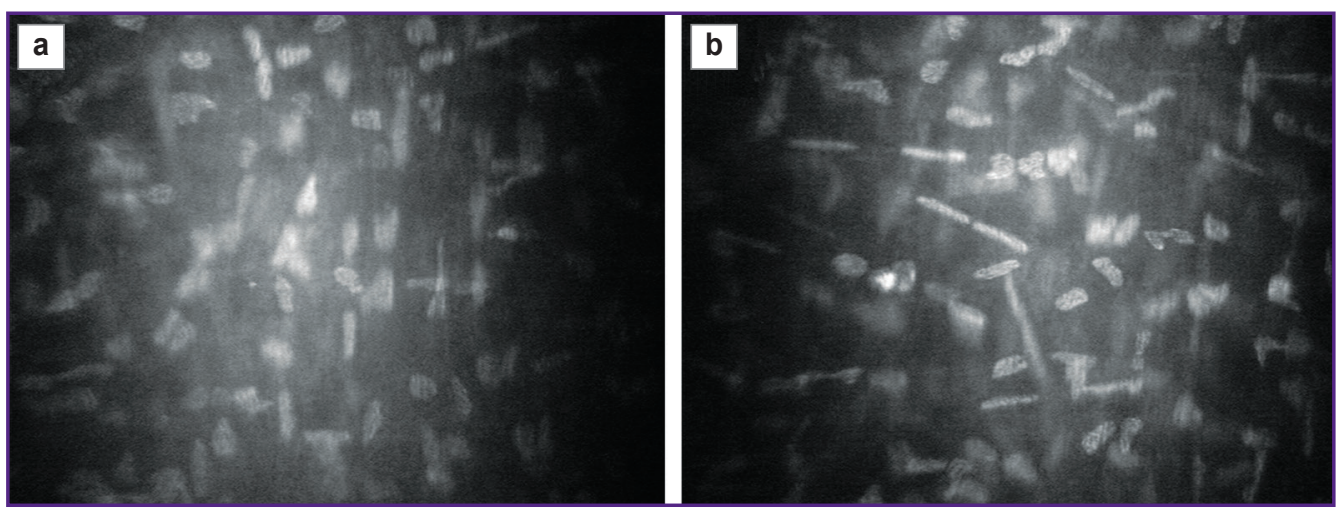

Figure 4. Vertical orientation of keratocyte nuclei (a), needle-shaped keratocyte nuclei in the posterior stroma (b); FOV $-300 \times 220 \mu \mathrm{m}$

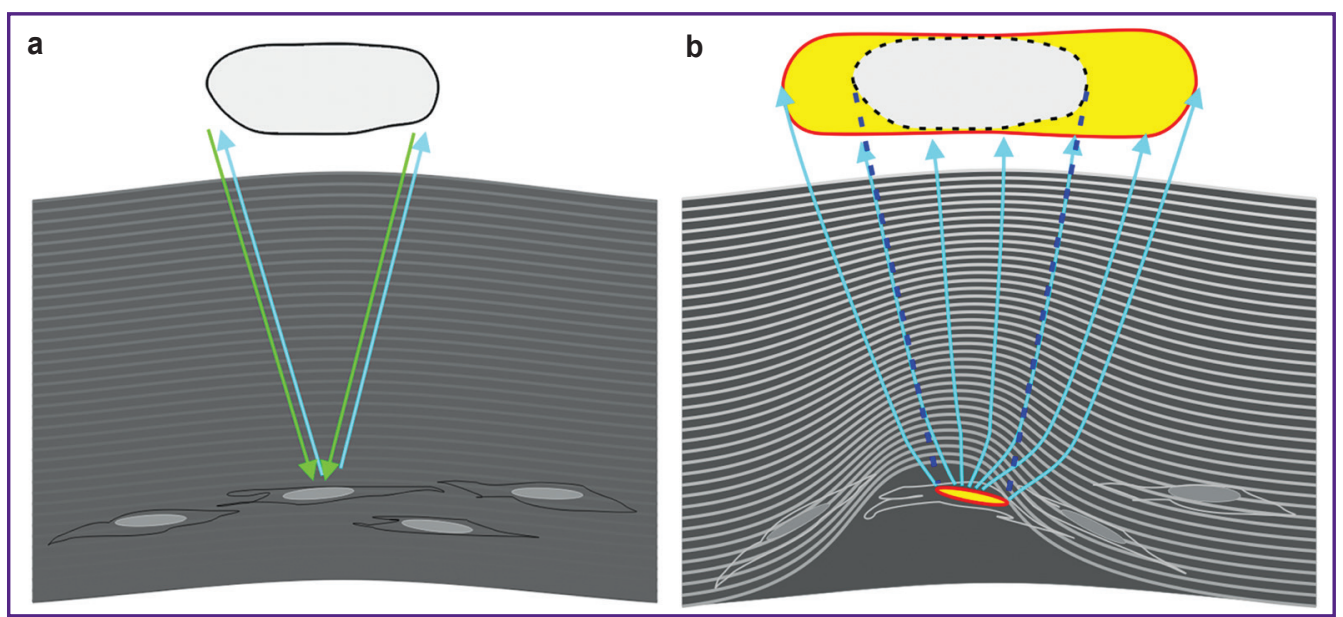

Figure 5. Confocal image formation in the norm (a) and in disturbed orientation of corneal stromal collagen lamellae (b)

The figure shows the imaginary distortion of formed stromal elements that occurs with the addition of a strong cylindrical lens in the form of a fold in collagen layers to the optical scheme of the confocal microscope; by the example of an imaginary keratocyte nucleus view we have demonstrated an imaginary visible nuclear elongation (yellow) 


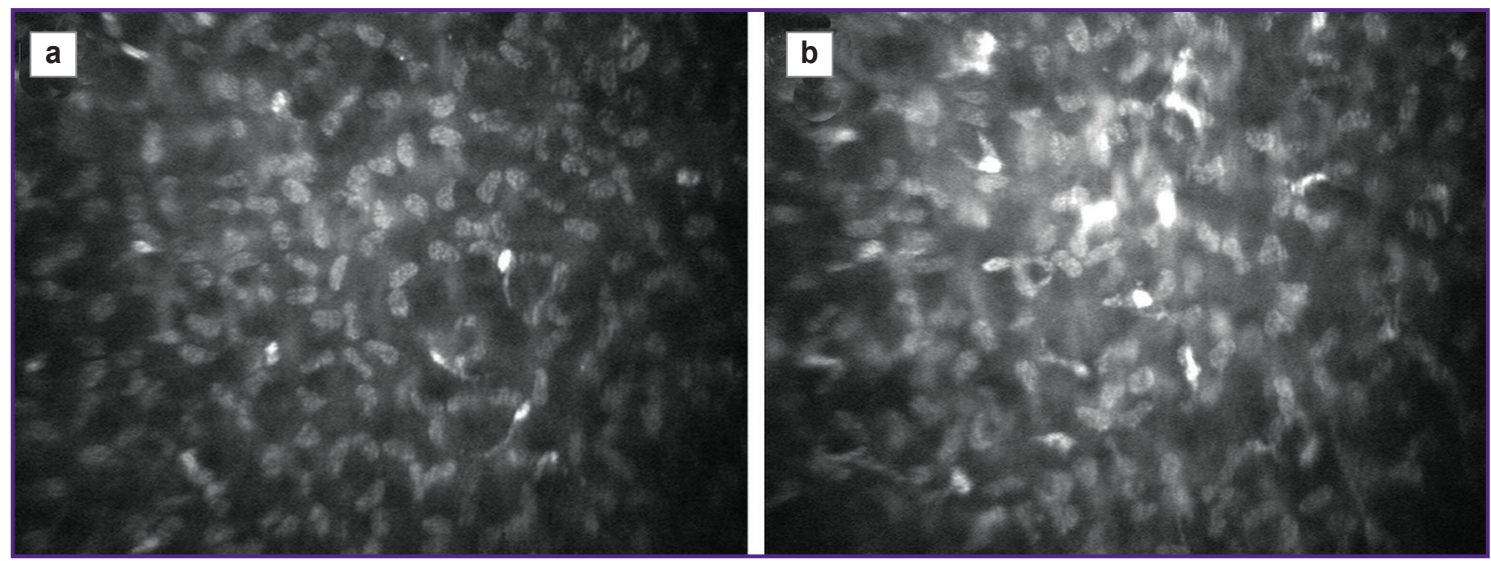

Figure 6. A microscopic picture of the anterior corneal stroma in the norm (a) and in stage I keratoconus (b); FOV $-300 \times 220 \mu \mathrm{m}$

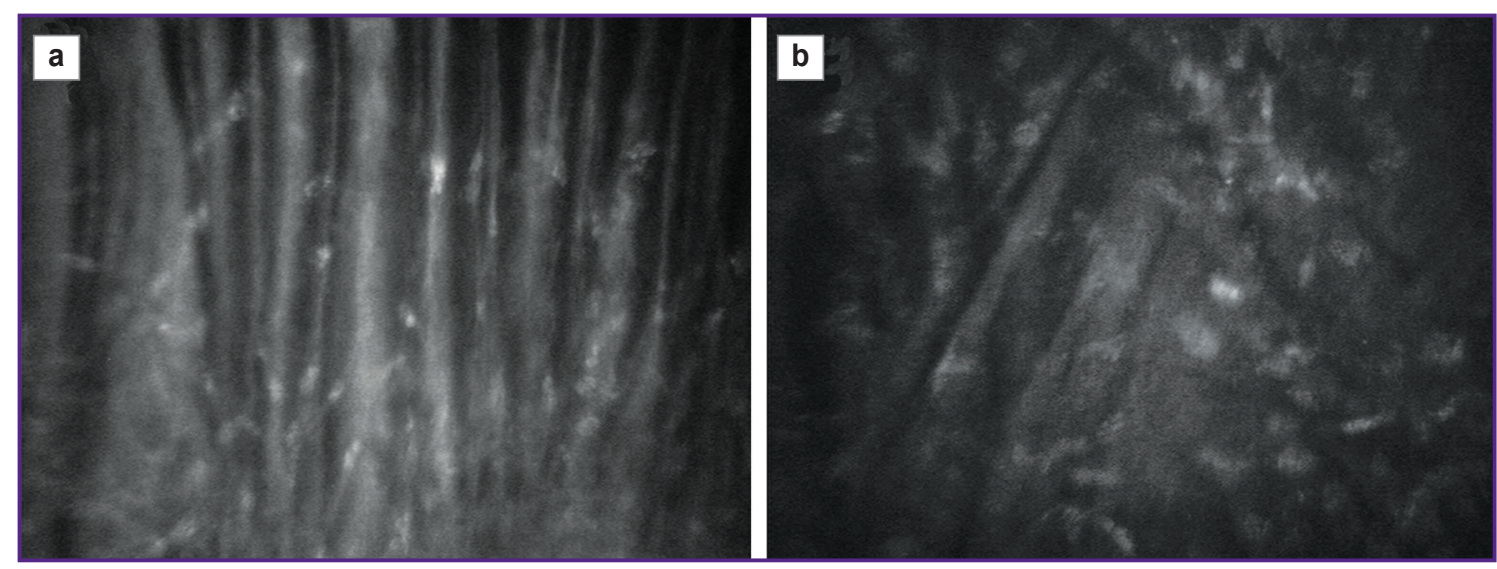

Figure 7. Microstrias in the posterior corneal stroma:

(a) vertically; (b) at an angle; FOV $-300 \times 220 \mu \mathrm{m}$

rarefaction", "firework", etc. [8-10]. However, confocal microscopy does not manage to reveal rarefaction signs in the front stroma layers, quite the opposite, the optical density of tissues and cell elements increases leading to light reflection growth. Microscopic treatment zone of high density form in the stroma, reflecting capacity of keratocyte nuclei increases causing optical homogeneity disturbance and the increase in a light scattering effect (Figure 6).

One of the most typical and specific manifestations of pathological processes in corneal stroma revealed by confocal microscopy (stage I-II keratoconus) is alternating black-white bands, the so-called microstrias. Generally, bands are vertically oriented, however, in some cases, microstrias can be arranged both: transversely and angle-wise (Figure 7). They are not seen at an initial stage in biomicroscopy, however, when fold formation results in sufficient light contrast, they are likely to be observed in the form of the so-called keratoconus lines. Keratoconus lines in certain cases are interpreted as "stress lines", "strain lines" or as a stroma strain sign resulted from the detachment and divergence of collagen plates [8-10]. Mastropasqua and Nubile [1] when describing a confocal microscopic picture in keratoconus have suggested that microstrias are the manifestation of the alteration and deformity of collagen plates, and can imply the formation of either microfolds or cracks in extracellular matrix.

According to our reckoning, if corneal confocal microscopy reveals microstrias and subsequently keratoconus lines visualized by biomicroscopy, it is due to further development of a pathological process and increased amplitude of corneal stromal folds. With stromal fold geometric amplitude increase, an absolute cylindric lens forms preventing the formation of confocal images of objects located behind the lens, in deeper layers. At the fold apex (at its certain stretching) it is possible to reflect the beams, which form a light band. There will be a dark band effect on fold limbs since the reflected beams bend and do not enter a microscope objective aperture (Figure 8).

The statement can be confirmed by histological findings. Histological sections of corneal discs obtained in penetrating keratoplasty demonstrate formed stromal folds spreading from the posterior layers to the middle ones. It also confirms the supposition that structural 


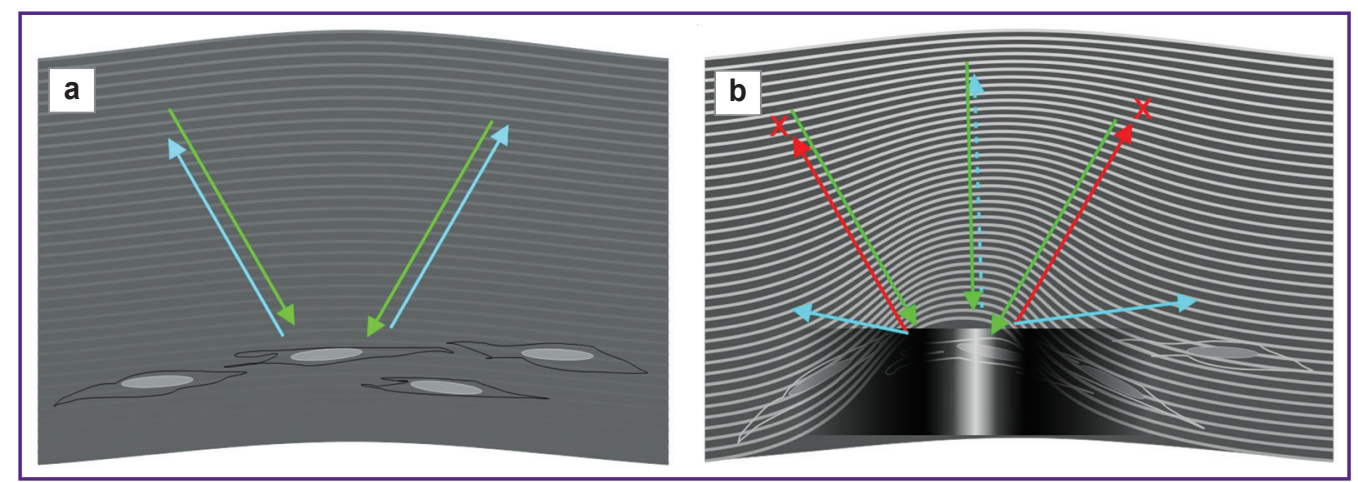

Figure 8. A principle of confocal image formation in the norm (a) and in the formation of corneal stromal steep folds (b)

Conventionally it is shown the occurrence of linear area of shading when the lamp beams are reflected from side surfaces of steep folds

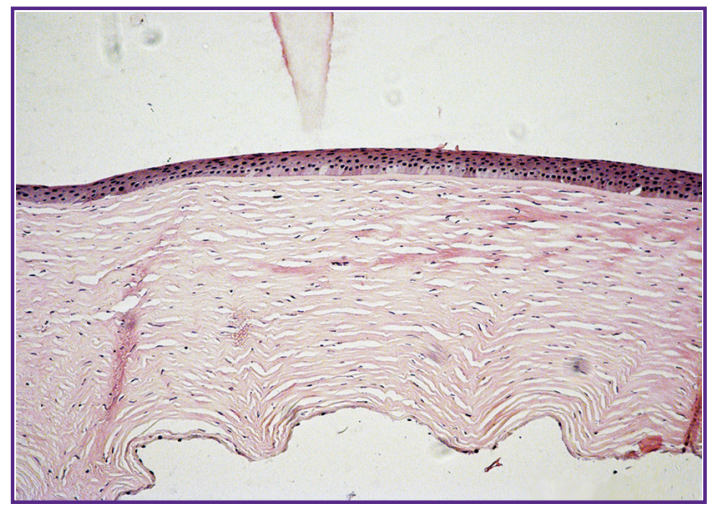

Figure 9. Stage III keratoconus

Nonuniform thickness of corneal stroma, wave-like posterior surface, fold formation in the posterior and the middle stroma layers, stroma hypocellularity; paraffin section; hematoxylin and eosin staining; FOV $-1000 \times 750 \mu \mathrm{m}$

disturbances in keratoconus are most expressed in the posterior stroma with their further extension to the middle and the anterior layers (Figure 9). From this point of view, it is easy to explain the symptom of the disappearance of keratoconus lines, which are seen in biomicroscopy, in slight compression on corneal surface. Compression makes folds smooth, decreases their amplitude resulting in optical phenomenon disappearance.

In keratoconus progression, pathological changes in epithelial layers and Bowman's membrane increase. In some cases, the defects can be registered using confocal microscopy (Figure 10 (a)).

If there occur Bowman's membrane defects, one can observe the activation of tissue adaptive and protective mechanisms being realized in two directions. Stromal defects close due to fibrocellular proliferation and fibrous tissue formation (Figure 10 (b)) that subsequently results in high reflective opaque areas registered in confocal microscopy in Bowman's membrane in advanced keratoconus.
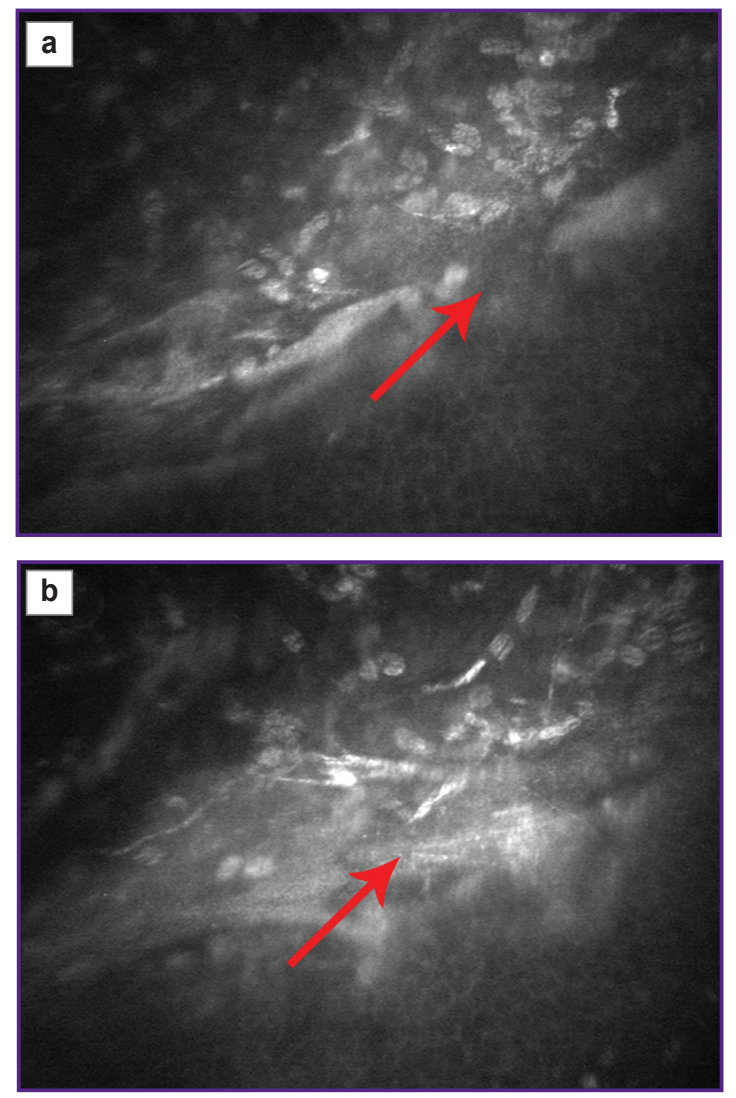

Figure 10. An arrows show Bowman's membrane defect (a) and fibrous tissue formation, substitution defect of Bowman's membrane (b); FOV $-300 \times 220 \mu \mathrm{m}$

On the part of epithelium in Bowman's membrane defect there are proliferation processes with cell edema and impaired morphology of epithelial layers that can be seen in a corneal histological section (Figure 11). In histological sections there are edematous cells of basal epithelium, spindle-shaped fibroblast-like cells of fibrocellular tissue substituting Bowman's membrane 


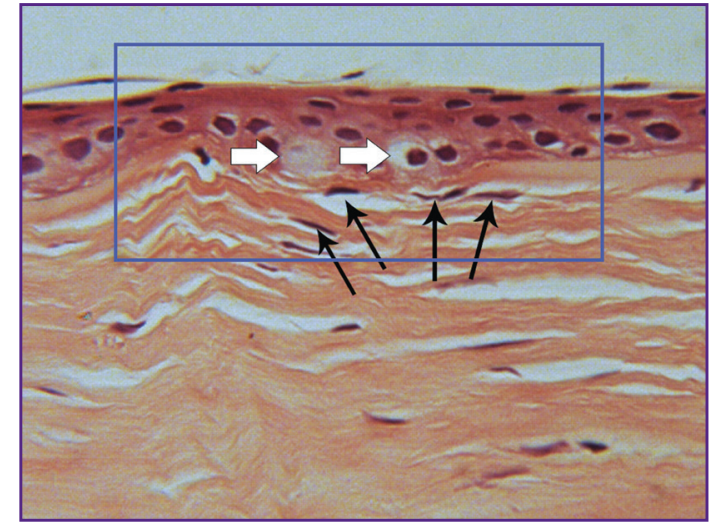

Figure 11. Substitution of Bowman's membrane defects by fibrocellular tissue (black arrows); edema of basal cells (white arrows)

Increased desquamation of superficial epithelial cells; paraffin section; hematoxylin and eosin staining; FOV $-250 \times 190 \mu \mathrm{m}$

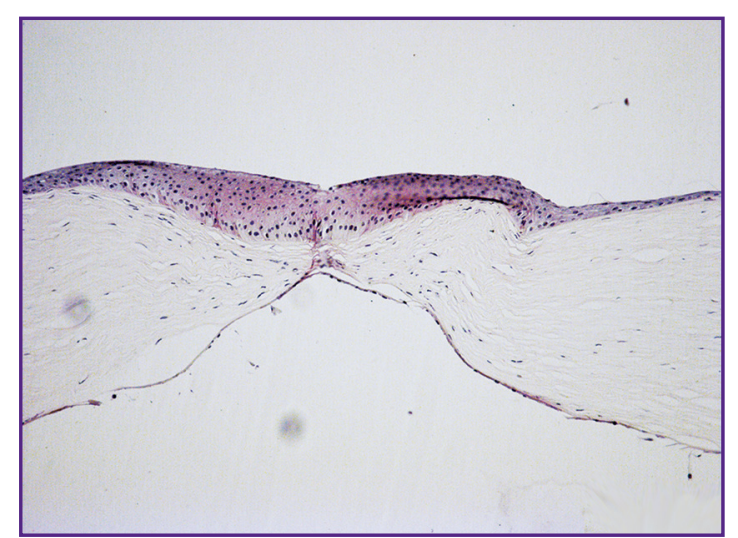

Figure 12. Stage IV keratoconus

Extreme thinning and cicatrization of corneal stroma, anterior epithelial hyperplasia, Descemet's membrane thinning and local separation of the posterior epithelium; hematoxylin and eosin staining; FOV $-1000 \times 750 \mu \mathrm{m}$ defects. There are squamous cells with a different desquamation degree on a corneal surface.

Many researchers carried out investigations to study histopathological changes in keratoconus. In particular, Sykakis et al. [11] in 2012 when describing morphological disturbances in keratoconus noted a positive correlation between the number of Bowman's membrane defects and corneal epithelial layer thickness. Epithelial "plugs" closing a defect of the epithelial part can form due to epithelial proliferation in Bowman's membrane defects.

Corneal histological section of the patient with stage IV keratoconus clearly demonstrates cornea adaptive protective mechanisms being switched in response to damage (Figure 12). There is no healthy corneal stroma in extreme thinning areas, it being substituted by fibrous tissue (scar tissue formation). On the epithelial part, a defect is closed by a thickened epithelial layer, and due to its marked proliferation, the anterior corneal defect is smoothed. There are Descemet's membrane thinning, separation and micro-ruptures. In the thinning and scar area, Bowman's membrane is missing, the anterior epithelium has hyperplasia, epithelial cells showing impaired alignment and differentiation.

Frequent findings in corneal histological sections in stage III-IV keratoconus are pathological changes of Descemet's membrane, its rupture, thinning, and separation. However, according to investigations, acute keratoconus by no means is caused by rupture. Confocal microscopy reveals closed defects of Descemet's membrane in patients with no acute keratoconus in their past history. The findings suggest that in a small defect protective tissue mechanisms manage to mobilize and close the defect. On stromal part there forms thick fibrous structureless tissue, which can partially be covered on the endothelial part due to migrating endothelial cells, as well as small, probably, transformed fibroblast-like cells (Figure 13).

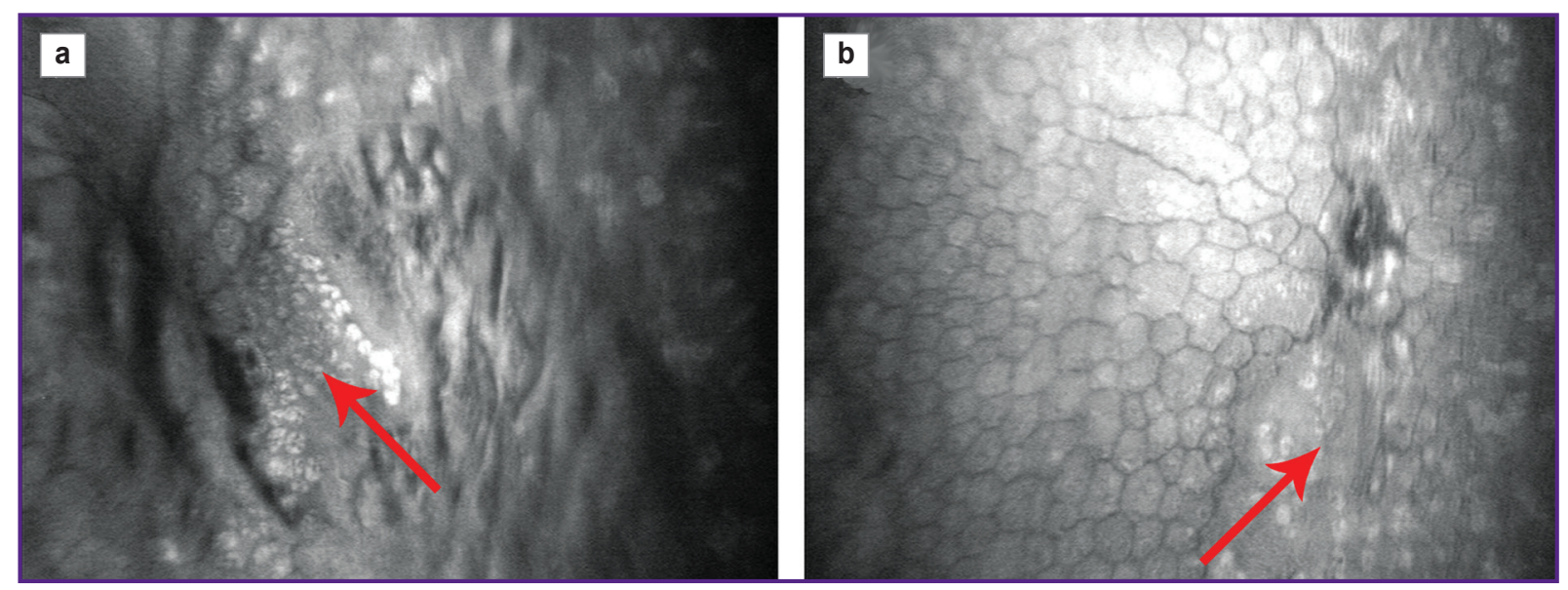

Figure 13. Cicatricial tissue (arrows) in the posterior corneal epithelium (a), partially covered by transformed fibroblast-like cells (b); FOV $-300 \times 220 \mu \mathrm{m}$ 


\section{Conclusion}

It is evident that genuine understanding of the alterations occurring at a tissue level, when the disease is progressing, is the key to interpret its pathogenesis. For the first time, there has been reconsidered the interpretation of corneal confocal biomicroscopic images due to light passage physics in biological tissues. By the example of morphological stromal changes in keratoconus there have been demonstrated the optical causes of distorted brightness and geometry of formed tissue elements, which previously were not recognized as the artifacts of the technique. The findings enable to assess corneal confocal microscopy informativity, suggest a certain interpretation of the findings and confirm it using a histological analysis.

Study funding. The study was carried out under research activities (State registration number 05112014-0031).

Conflicts of Interest. The authors declare no conflicts of interest related to the present study.

\section{References}

1. Mastropasqua L., Nubile M. Confocal microscopy of the cornea. USA: SLACK Incorporated; 2002.

2. Handbook of biological confocal microscopy. Pawley J.B. (editor). Springer US; 2006, https://doi. org/10.1007/978-0-387-45524-2.
3. Minsky M. Microscopy apparatus. US patent 3013467A. 1961.

4. Shchelokov R.V., Yatsyshen V.V. Ellipsometricheskiy metod $v$ diagnostike sred so slozhnoy molekulyarnoy strukturoy (rogovitsa glaza). V kn.: X Mezhdunarodnaya shkola seminar "Elektrodinamika $i$ tekhnika SVCH, $\mathrm{KVCH} i$ opticheskikh chastot" [Ellipsometric method in diagnosing the media with complex molecular structure (eye cornea). In: X International school-seminar "Electrodynamics and technology of SHF, EHF and optical frequencies"]. Moscow; 2002; vol. 10(X); 2(34): 246.

5. Tuchin V.V. Light scattering study of tissues. Uspekhi fizicheskikh nauk 1997; 167(5): 517-539.

6. Egorova G.B., Rogova A.la., Mitichkina T.S. Diagnostic value of confocal microscopy in primary corneal ectasia. Vestnik oftalmologii 2012; 128(6): 25-29.

7. Lushnikov E.A., Abrosimov A.Yu., Gabay V.L., Saenko A.S., Dorosevich A.E. Gibel kletki (apoptoz) [Cell death (apoptosis)]. Moscow: Meditsina; 2001.

8. Keratoconus and keratoectasia: prevention, diagnosis and treatment. Wang M. (editor). USA: SLACK Incorporated; 2010.

9. Kivaev A.A., Babich G.A., Abugova T.D. Dynamics of biomicroscopic changes in keratoconus cornea. Oftalmologicheskiy zhurnal 1979; 34(4): 217-218.

10. Kasparova E.A. Diagnosis and treatment of early keratoconus. Glaz 2001; 2: 35-40.

11. Sykakis E., Carley F., Irion L., Denton J., Hillarby M.C. An in depth analysis of histopathological characteristics found in keratoconus. Pathology 2012; 44(3): 234-239, https://doi. org/10.1097/pat.0b013e3283511b42. 